\title{
Narrativa
}

narrativa

Nuova serie

$42 \mid 2020$

Letteratura e economia nell'Italia degli anni Duemila

\section{Vexata Lectio: la Lehman Trilogy di Stefano Massini per ri-spettare i nostri giorni}

\section{Maurizio Rebaudengo}

\section{(2) OpenEdition}

\section{Tournals}

Edizione digitale

URL: https://journals.openedition.org/narrativa/293

DOI: $10.4000 /$ narrativa.293

ISSN: 2804-1224

\section{Editore}

Presses universitaires de Paris Nanterre

\section{Edizione cartacea}

Data di pubblicazione: 1 décembre 2020

Paginazione: $79-91$

ISBN: 978-2-84016-381-7

ISSN: $1166-3243$

\section{Notizia bibliografica digitale}

Maurizio Rebaudengo, «Vexata Lectio: la Lehman Trilogy di Stefano Massini per ri-spettare i nostri giorni», Narrativa [Online], 42 | 2020, online dal 01 décembre 2021, consultato il 08 décembre 2021. URL: http://journals.openedition.org/narrativa/293 ; DOI: https://doi.org/10.4000/narrativa.293

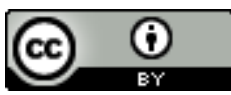

Narrativa est mise à disposition selon les termes de la Licence Creative Commons Attribution 4.0 International. 


\section{Vexata Lectio: la Lehman Trilogy di Stefano Massini per ri-spettare i nostri giorni}

\section{RiASSUNTO}

Il saggio propone una riflessione sulla Lehman Trilogy di Stefano Massini e sul romanzo/ ballata che l'ha seguita - Qualcosa sui Lehman - spingendosi fino a riflettere sulla pandemia che ha travolto il mondo intero nel 2020 e criticando in particolare la speranza di un ritorno alla normalità. La crisi finanziaria, cominciata col tracollo della celebre banca americana di cui Massini ricostruisce la genesi come fosse un mito moderno, diventa una sorta di prologo alla crisi sanitaria che il mondo sta attraversando ed è interpretabile come una concreta rappresentazione degli orizzonti economici globali e globalizzati.

\section{RÉSUMÉ}

Cet article propose une réflexion sur la Lebman Trilogy de Stefano Massini et le roman qui l'a suivie - Qualcosa sui Lehman - et il va jusqu'à évoquer la pandémie qui a balayé le monde en 2020, en critiquant notamment l'espoir d'un retour à la normale. La crise financière qui a commencé avec l'effondrement de la célèbre banque américaine, dont Massini reconstitue la genèse comme s'il s'agissait d'un mythe moderne, devient une sorte de prologue à la crise sanitaire que traverse le monde et peut être interprétée comme une représentation concrète des horizons économiques mondiaux et globalisés.

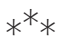


Ancora non sappiamo quali potranno essere gli esiti di una auspicabile autoriforma del capitalismo, unica condizione per evitare quella che è da sempre l'antitesi di ogni progetto riformista ovvero la rivoluzione, generalmente cruenta e indiffereñiata ${ }^{1}$.

Con la pandemia del Covid-19 ancora in corso, scrivere di un testo teatrale - ampliato poi in romanzo/ballata dal titolo Qualcosa sui Lehman ${ }^{2}$-, che ha indotto spettatori e lettori nel mondo a riflettere sui meccanismi produttivi della crisi finanziaria, scaturigine di quella economica, cominciata nel settembre del 2008 col fallimento della banca d'investimenti Lehman Brothers \& Co., dovrebbe servire - almeno si spera, nelle intenzioni di chi redige queste righe a riflettere sulla coazione al fallimento dell'Occidente avanzato, fino ad ora incapace di dare al lemma sviluppo significati concreti che divergano dal sereno e gaudente orizzonte di edonistico sfruttamento (di persone, luoghi e tempi), per quanto proclami e programmi dei decisori politici illudano talvolta a sperare altrimenti. Dovrebbe servire: mai modo condizionale fu più appropriato, appunto.

La relazione tra la cosiddetta Grande Recessione - la più grave crisi economica, o almeno di maggior durata, dai tempi di quella del 1929 -, causata dalle drammatiche ricadute sull'economia reale delle fallimentari speculazioni finanziarie, andate poi pure impunite ${ }^{3}$, e le fragilità di sistema mai sanate in questi anni ${ }^{4}$, avvolte dalla pesante coltre pandemica, è stata subito individuata dagli esperti del settore, facendo emergere in modo violentemente impietoso lo stringente vincolo tra due necessità tra loro ormai conflittuali nel capitalismo avanzato, globalizzato e postmoderno (o, per meglio dire, post-reale): la tutela del diritto alla salute, biologicamente universale e naturale (affidato ai sistemi

1. Ronconi Luca, Prefazione in Massini Stefano, Lebman Trilogy, Torino, Einaudi, 2014, p. VIII.

2. Massini Stefano, Qualcosa sui Lehman, Milano, Mondadori, 2016. Cfr. per la “pseudoversificazione” D'ONGHIA Luca, "Esperimenti linguistici nel teatro italiano degli ultimi vent'anni”, in Frigorilli Maria Cristina, Vianello Daniele (a cura di), La commedia italiana. Tradizione e storia, Bari, Edizioni di Pagina, 2018, pp. 248-249.

3. Cfr. Eisinger Jesse, "Why Only One Top Banker Went to Jail for the Financial Crisis", The New York Times Magazine, 30 aprile 2014, https://www.nytimes. com/2014/05/04/magazine/only-one-top-banker-jail-financial-crisis.html.

4. Cfr. Luna Riccardo, "Perché siamo tutti figli di Lehman Brothers", Agi.it, 14 settembre 2018, https://www.agi.it/blog-italia/riccardo-luna/lehman_brothers_ italia_cambiamento_dati-4378170/post/2018-09-14/. 
pubblici di welfare, non sempre collimanti con gli interessi privati) $)^{5}$, e la tutela del diritto al lavoro, ridotta a garantire la sopravvivenza materiale quotidiana (senza peraltro riuscirci sempre), venendo meno il margine di un indotto bisogno al consumo diffusamente accolto come ormai naturale anch'esso ${ }^{6}$.

[...] è sacrosanto che la fatica del lavoro pretenda un contraccambio: è l'sstinto dell'animale che lo reclama in noi. Silenziare questo disagio non significa sopprimerlo. Né ci basta abbeverarci alla sempre provvida sorgente dei buoni propositi e delle dilazioni, illusorie quanto ridicole: nella Legge esiste, ad esempio, il cosiddetto "privilegio dei lavoratori". Niente di più che un sano principio per cui, in caso di fallimento i primi a essere ripagati dovranno essere i dipendenti non remunerati, fieri d'essere in cima alla lista dei creditori. Ovviamente questo avverrà in futuro, dopo l'intervento della magistratura [...]. In altri termini, per capirci, mozzi e marinai possono dormire sonni tranquilli: se il loro armatore andrà in rovina, i pezzi della nave toccheranno per primi all'equipaggio. Un giorno, naturalmente. Prima o poi. Intanto lavorate sereni'

Nelle parole di Stefano Massini, il lavoro, da ostentazione pubblica (l'etimo di "professione": profiteor $)^{8}$ per ottenere credibilità sociale, si è trasformato in mera "occupazione" (da ob-capere) come etimologico controllo ostile "di un territorio, letteralmente sottratto alla potestà altrui", generando per il soggetto

5. Cfr. Zerhouni Will, Nabel Gary J., Zerhouni Elias, "Patents, economics, and pandemics", Science, vol. 368, n. 6495, 5 giugno 2020, https:/ / science.sciencemag.org/ content/368/6495/1035.

6. Per le conseguenze finanziarie della pandemia e l'innescarsi del domino vizioso pandemia-economia finanziaria-economia reale, cfr. CiCCARELLI Roberto, "Il Coronavirus come la Lehman Brothers: il capitalismo è entrato nella fase 'orso", il manifesto, 9 marzo 2020, https://ilmanifesto.it/bagno-di-sangue-in-borsa-e-il-crollo-peggiore-dal-2008/. Per un chiaro e sintetico confronto (anche grafico) tra le due situazioni da uno specialista del settore, cfr. Szopo Peter, "Lehman Brothers and Coronavirus. A (Preliminary) Tale of Two Crises”, Erste Asset Management Blog, 30 marzo 2020, https://blog.en.erste-am.com/ lehman-brothers-and-coronavirus-a-preliminary-tale-of-twocrises/; STRAUSS KAHN Marc Oliver, "Can we compare the COVID-19 and 2008 crises?", New Atlanticist, 5 maggio 2020, https://www.atlanticcouncil.org/blogs/new-atlanticist/can-we-compare-the-covid-19and-2008-crises/. Già il 29 febbraio 2020, l'Osservatorio dei Conti Pubblici Italiani della Università Cattolica di Milano, diretto da Carlo Cottarelli, pubblicava un'analisi sulle simulazioni dei possibili scenari economici causati dal Covid (si ricordi un dato cronologico importante: a quella data la pandemia non era ancora stata dichiarata tale dall'OMS, e non se ne conosceva ancora bene la letalità), prevedendo un inevitabile shock sull'economia globale, non ancora però quantificabile (FRATTOLA Edoardo, "Che effetti può avere una pandemia sull'economia mondiale?”, OCPI, 29 febbraio 2020, https://osservatoriocpi.unicatt.it/ cpi-archivio-studi-e-analisi-che-effetti-puo-avere-una-pandemia-sull-economia-mondiale).

7. Massini Stefano, Lavoro, Bologna, Il Mulino, 2016, pp. 53-54.

8. Ibid., p. 29.

9. Ibid., p. 32. 
lavorante una espropriazione della coordinata temporale, non sanabile con l'ormai diffuso smart working. I vocaboli italiani più diffusi per esprimere la retribuzione del lavoro - ricompensa, salario e stipendio ${ }^{10}$ - sono la soddisfazione di un "istinto animale", che non dà alcuna garanzia, però, nei casi di improvvisa e non sempre lecitamente ragionevole sospensione o interruzione dell'equo scambio: in quei casi, allora, si svela la natura contemporanea del lavoro, una pro-messa (mettere avanti, non certo a difesa di), la dilazione della sicurezza e dei diritti, in nome di un vantaggio momentaneo ed illusorio (intanto ti sfrutto: poi, quando sarà possibile, ti darò quanto ti spetta).

Nella Lehman Trilogy, e ancor più nel romanzo, l'autore esamina il progressivo rarefarsi del lavoro nel suo concreto rapporto con la materia prima, pressoché ignorata da chi ne trae realmente profitto: nel capitolo I della parte II di Qualcosa sui Lehman - The Black Hole ${ }^{11}$ - il cosiddetto "braccio" dei tre fratelli fondatori, Emanuel, accompagnato dall'adrenalinico nipote Dawid, si allontana da New York per ispezionare i filoni carboniferi delle miniere Wilcock, traendone così la positiva motivazione all'investimento; nel capitolo IV (sempre parte II) di Qualcosa sui Lehman - Oklahoma ${ }^{12}$ - i due fratelli sopravvissuti (Emanuel e Mayer) si recano insieme al nipote Dreidel in Oklahoma dal proprietario della Spencer Oil, per esplorare le possibilità di un accordo in esclusiva, che dia loro il controllo anche del mercato petrolifero. Ma trattasi di meri intermezzi narrativi, assenti del tutto da Lehman Trilogy e anche dalla parte III di Qualcosa sui Lehman, che non alterano per nulla il distacco tra l'astratta filiera calcoloquotazione-profitto e la sua origine in natura.

10. “re-con-pensare, ovvero letteralmente 'porre un contrappeso' [...] la parola 'salario' rimanda alla misura del compenso dei mercenari ([...] stips, da cui 'stipendio', ovvero pesare con spiccioli)" (ibid., pp. 47-48).

11. Massini Stefano, Qualcosa sui Lehman, cit., pp. 185-194.

12. Ibid., pp. 215-228. In Lebman Trilogy l'interesse per il petrolio è delegato a pochi versi, dai quali si ricava che a ispezionare i pozzi di petrolio sono sempre i due fratelli superstiti, ma in compagnia di Philip, figlio di Emanuel e successivo king maker della società, senza il divertente siparietto con l'insopportabile cane del padrone di casa, il proprietario della Spencer Oil, e la conclusiva esplosione dei pozzi, appiccata dall'incontenibile rabbia di Dreidel: "Sarà per questo che/ l'idea è piaciuta subito/ sia a Mayer che a Emanuel/ quando Philip li ha portati laggiù/ in Oklahoma/ e poi in Ontario/ a vedere i pozzi in azione:/ trivelle agitatissime/ rumore da non dire/ schizzi neri fino al cielo/ rubinetti da giganti/ e ferro/ e tubi/ e condutture./ Insomma, il petrolio c'è: si vede" (ID., Lehman Trilogy, cit., p. 140). Si ricordi anche il viaggio del medesimo trio a Baton Rouge, in Louisiana, per mettere le mani sul commercio dello zucchero [in questo caso, a combinare il disastro è la schifata reazione di Dreidel all'assaggio del tanto decantato dolcificante (ID., Qualcosa sui Lehman, cit., pp. 104-108)]. 
Dal momento in cui la sede della società trasloca dalla prima capitale della Confederazione sudista, Montgomery in Alabama, a New York, la Lehman Brothers, perno della ricostruzione successiva alla Guerra Civile ${ }^{13}$, abbandona sempre più la familiarità tattile con la fonte di reddito - il cotone -, compiendo una operazione cruciale nella evoluzione dell'economia non solo americana: la rarefazione di senso - le merci e le materie prime, il dato concreto della stipula - nel segno (i titoli azionari e obbligazionari a sostituire e nascondere il lavoro, le condizioni precarie e anche sporche della sua effettuazione) e i suoi frutti (banca di investimenti: compravendita di danaro, bene che non si vede, ma esiste perché dato numerico parte di un calcolo). Agli oggetti, quindi, si sostituiscono delle parole, che col tempo la tecnologia ridurrà a serie numeriche governabili tramite algoritmi, proiettate su schermi che riempiono la sala delle contrattazioni nell'ultima fase della Lehman Brothers.

Il brulichio verbale, il frastuono della retorica con cui nelle metropoli si creano le reputazioni e le relazioni, fondamentali per plasmare il collante primo degli accordi economici, la fiducia reciproca, vengono trasposti in entrambi i testi nelle insistite ripetizioni a blocchi di versi ricorrenti come da epica omerica $^{14}$, a volte a uso mnemotecnico a volte per indicare l'ostinata volontà con cui i fratelli Lehman, e i loro discendenti, perseguono - e raggiungono - i loro obiettivi, economici quanto matrimoniali. Sono l'elemento di più eclatante novità colto dai fratelli al loro arrivo nella capitale economica degli USA: abituati al rigore paterno di stampo rabbinico, ancorato alle cose, per cui già una stoffa è una impropria avventatezza ${ }^{15}$, che deraglia dai mitzvot a cui un vero ebreo dovrebbe attenersi, i Lehman (i tre fratelli, in definitiva, passano dalle campagne bavaresi al benestante ma provinciale Sud confederato) comprendono fin da subito di essere capitati in un mondo in cui il dominio delle parole - e la loro manipolazione, se necessario - è alla base della integrazione in una società libera per chi sappia fruirne le possibilità.

13. Cfr. Cohen Michael R., Cotton Capitalists. American Jewish Entrepeneurship in the Reconstruction Era, New York, New York University Press, 2017, passim.

14. Cfr. Di Paolo, Paolo, "Segui i soldi e troverai l'America: 'Qualcosa sui Lehman', di Stefano Massini”, La Stampa, 8 ottobre 2016, https://www.lastampa.it/ tuttolibri/2016/11/08/news/segui-i-soldi-e-troverai-l-america-qualcosa-sui-lehmandi-stefano-massini-1.37601203.

15. "LA PAROLA DI UN UOMO,/ FIGLIO CARO, È INCISA SU PIETRA;/ LA PAROLA DI UNO SCIOCCO/ E SCRIT'TA SULLA STOFFA": così scrive su di un biglietto il patriarca Abraham Lehman, indirizzato in busta chiusa al figlio maggiore "Herr Heyum Lehman” (Massini Stefano, Qualcosa sui Lehman, cit., p. 41). 
Non a caso, sia nel testo teatrale sia nel romanzo/ballata, la narrazione comincia con il capitolo Luftmensch, un vocabolo yiddisch il cui significato è "uomo d'aria", senza però alcuna allusione all'inconsistenza: può essere usato per i sognatori, ma soprattutto per coloro che non hanno radici, che vivono dunque staccati da terra ${ }^{16}$. Al suo arrivo al molo n. 4 di New York, Heyum Lehmann accetta l'alterazione d'identità in Henry Lehman per l'impossibilità di far comprendere l'inusuale fonetica all'ufficiale preposto alla registrazione dei nuovi arrivati, ma una volta fissato quel cognome, i fratelli sono ossessivamente pignoli nello scandirlo correttamente e nell'affiggerlo, perché è quel cognome - e non l'originario - a costituire il veicolo della loro fortuna economica.

La metaforica cancellazione di una identità meramente anagrafica non comporta, però, la cancellazione dell'identità culturale e religiosa, che anzi è un pilastro portante della scrittura di Massini, pur non essendo egli ebreo ${ }^{17}$.

Nella vicenda dei Lehman la distanza geografica dal rigido moralismo paterno - che fa sentire la sua presenza con sporadiche quanto sibilline massime - permette alla dinastia di ebrei tedeschi emigrati nel Nuovo Mondo di non percepire l'etica loro propria come ostacolo, continuando comunque a ricorrere all'autorità spirituale di un rabbino per le cruciali scelte strategiche, e trasformando anzi una tradizione in un incentivo a osare. Ne è un esempio lampante un episodio collocato all'inizio di Qualcosa sui Lehman, nel capitolo II della prima parte (Gefilte Fish), in cui il neoarrivato Henry Lehman segue il consiglio degli amici tedeschi, suoi ospiti, a consultare Rab Kassovitz, che gode fama di oracolo, un Tiresia talmudico ${ }^{18}$ : accolto il giovane migrante con irridente sgradevolezza, lo conduce maieuticamente a comprendere il significato di una metafora ittica:

Se il pesce - che è giunto a Nuova York dall'immenso mare - / si infilasse da quel mare dentro un fiume/ e dal fiume in un canale/ e dal canale in un lago/ e dal lago in uno stagno/ adesso io vi domando:/ non riuscirebbe davvero quel

16. Il grande critico George Steiner si autodefiniva tale: SIEGEL Richard, SOFER Tamar (a cura di), The Writer in the Jewish Community. An Israeli-North American Dialogue, Cranbury-London-Missisauga, Associated University Presses, pp. 124-125.

17. Cfr. Trevale Annamaria, "Premio Campiello 2017 - Intervista a Stefano Massini", Sulromanzo.it, 10 luglio 2017, http:/ / www.sulromanzo.it/blog/premio-campiello-2017intervista-a-stefano-massini. Prima della consacrazione con la Lehman Trilogy, Massini aveva già trattato della cultura ebraica in altri due testi teatrali: Processo a Dio e La fine di Shavnoth, in Una quadrilogia. L'odore assordante del bianco, Processo a Dio, Memorie del boia, La fine di Shavnoth, Roma, Edizioni Ubulibri, 2006, poi Torino, Einaudi, 2017.

18. "Quel vecchio rabbino, con tutto che ha gli occhi storti,/ riesce a guardare dove tu non vedi,/ e a dirti chi sarai in quest'altra vita" (MAssini Stefano, Qualcosa sui Lehman, cit. p. 16). 
pesce/ a girare l'America in lungo e in largo?/ Non gli è proibito: l'acqua scorre ovunque./ Il pesce deve solo ricordare che lui vive sommerso/ e se ne esce, semplicemente muore ${ }^{19}$.

Fin dall'inizio della vicenda, quindi, il percorso di una dinastia diventa paradigma di un passaggio dalla interculturalità alla transculturalità, che nello sviluppo narrativo sarà legato al passaggio dall'economia reale all'economia finanziaria: l'integrazione e l'interazione culturali e la capacità di influire in modo determinante sul contesto di arrivo, senza venire meno agli obblighi della comunità, l'appartenenza alla quale - però - sbiadisce con la progressiva perdita di attaccamento alle radici (tedesche o confederate che siano) e la conseguente difficoltà a riconoscersi nei suoi fondamenti etici. Ne è avvisaglia un episodio del capitolo XI, prima parte, di Lehman Trilogy (Reb Shalon) e XVIII - sempre prima parte - di Qualcosa sui Lehman (Hasele): sulla veranda di casa, a Montgomery, la moglie di Mayer, Babette Newgass, racconta ai due figli Sigmund e Arthur una favola: un tempo un rabbino di nome Reb Lashon, o il "Rabbino Lingua", parlava così velocemente da non riuscire a farsi capire, destando anzi l'ilarità dei fedeli nella preghiera serale al Tempio. La sua richiesta di poter parlare in modo comprensibile viene finalmente ascoltata, ma i fedeli a quel punto lo abbandonano, perché trovano i suoi precetti terribilmente noiosi ${ }^{20}$.

Nei due testi, l'autore alterna nella titolazione dei capitoli la lingua ebraica propriamente detta, l'yiddisch e inglese, ma con pesi diversi: se in Qualcosa sui Lehman il primo titolo in inglese lo troviamo solo al capitolo I della parte II (The Black Hole), in Lebman Trilogy (che seppur pubblicato prima del romanzo, ne costituisce una sintesi in quanto composto successivamente) ${ }^{21}$ già nel capitolo IV della parte I troviamo l'inglese (la riproduzione di un titolo di giornale: Hannukah, or What happened after fire), seguito da Something about New York (capitolo VIII; in Qualcosa sui Lehman: Libe in New York, capitolo XIII) e A little glass (capitolo X; Qualcosa sui Lehman: A glaz, biker, capitolo XVI), mentre in Qualcosa sui Lehman si aggiunge il capitolo XII, Sugarland (non presente nel testo teatrale). Nelle altre due parti dei testi, la scansione dei capitoli è data dall'alternarsi di ebraico, inglese e yiddisch, con una precisa attribuzione funzionale: l'ebraico, la lingua della cultura, è adoperata per riferirsi a occasioni e rituali o a personaggi veterotestamentari, assunti a paradigmi etici anche nell'agire professionale;

19. Ibid., pp. 20-21.

20. Ibid., pp. 161-162; ID., Lehman Trilogy, cit., p. 76.

21. Trevale Annamaria, "Premio Campiello 2017 - Intervista a Stefano Massini", cit. 
l'inglese per manifestare l'avvenuta acquisizione della civiltà d'accoglienza; l'yiddisch, come lessico familiare, residuo larario di un passato sopravvissuto solo più lessicalmente, sparisce completamente nella III parte di entrambi i testi² ${ }^{22}$.

Per i capitoli finali, l'autore intitola Monday Lunch l'Epilogo in Lehman Trilogy, ma senza specificazioni ulteriori in Qualcosa sui Lebman. Massini non mette in scena la bancarotta della gloriosa impresa commerciale e finanziaria che ha segnato la cultura mondiale del ' 900 (dal Canale di Panama alla motorizzazione di massa, dall'avvento dei mass media e del cinema sonoro al trasporto aereo, dalla diffusione dei beni di consumo allo sviluppo dell'informatica), ma assegna ai testi una conclusione di inequivocabile significato aristotelicamente tragico: raccoglie infatti attorno a un tavolo, come un abituale consiglio di amministrazione, i membri defunti della famiglia, che non controlla più la società, essendo ormai gestita nella attività principale - il trading - dal figlio di un gestore greco di una tavola calda nel Nebraska (anch'egli dal cognome cambiato) e dal figlio di un artigiano ungherese, fabbricante di lampade da tavolo. Queste ombre ricevono la comunicazione telefonica della morte della società, preparandosi quindi per il rito della Shivà, il periodo di lutto di sette giorni per la morte di parenti stretti di primo grado.

Questa parola ebraica intitola tre capitoli, in ciascuna parte di entrambe le opere (unico titolo a ricorrere in tutte e tre le parti): nella parte I per la morte

22. Oltre a Luftmensch e Gefilte Fish (presente solo in Qualcosa sui Lehman), per l'yiddisch, troviamo nella parte I di Lehman Trilogy: Bulbe ("patata", il soprannome di Mayer Lehman: capitolo III), Shpan dem loshek!, Kish Kish, Kiddushin, Der Boykhreder, solo in Qualcosa sui Lehman: Schmuck! (vocabolo entrato nell'uso comune dell'inglese colloquiale), Süsser (parola anche tedesca), Schmaltr, Shvarts zup (la "zuppa nera", a indicare il caffè); nella parte II di LT: Der bankir bruder, Der kartyoßhnik, Una tsvatsinger, Nisht trinken bronfn!, Der akrobat, solo in Qualcosa sui Lehman: Familie-Lehmann, Der terbyalant Dawid, Tsu fil rash, Der stille pakt, e una incursione nel tedesco propriamente detto: Eine Schule für Sigmund. Per l'ebraico: nella parte I di Lehman Trilogy si trovano Chametr, Shivà; in Qualcosa sui Lehman, in aggiunta, Shammàsh, Hanuke.kh, Yom Kippur, nella parte II di LT: Shavnoth, Bar-Mitzvah, Milah, e così pure in Qualcosa sui Lebman; nella parte III di Lehman Trilogy: Noach, Golyat, Shivà, Yonah, Migdol Bavel, Egel haZahav; solo in Qualcosa sui Lehman: Yitzach (per i significati dei termini ebraici e yiddisch cfr. Qualcosa sui Lehman, cit., pp. 795-803). Per l'inglese, invece, oltre a quanto già scritto per la parte I di entrambi i testi, nelle parti II troviamo: in Lehman Trilogy: Wall Street, Horses, Model T; in Qualcosa sui Lehman: The black hole, Henry's boys, Oklahoma, Studebaker, Stock Exchange, United Railways, Looking for Ewa, Olympic Games, The golden Philip, Pineapple juice, Babes in Toyland, Battlefield, A lot of words. Nelle parti III, invece: in Lehman Trilogy: Rainbow, I have a dream, Twist, Squash, Monday Lunch; in aggiunta in Qualcosa sui Lehman: The Arthur Method, NOT, One William Street, Roaring Twenties, Peloponnesus, A flying acrobat, Business in Soho, The fall, The Universal Flood, King Kong, Melancholy Song, Einstein or the genius, Technicolor, Enemies within, Saturday Game Show. 
improvvisa di Henry Lehman, a 33 anni di febbre gialla a New Orleans; nella parte II per la morte di Mayer Lehman, vegliato dal fratello più anziano Emanuel; nella parte III per la morte di chi ha introdotto la Lehman Brothers nel Novecento, Philip Lehman, figlio di Emanuel, un passo però in cui si tiene a ricordare che ormai (nel 1947) le usanze non vengono rispettate alla lettera né la banca viene chiusa per lutto ("Ora che sono sangue americano, i Lehman/ chi se li ricorda i riti dell'Europa?") ${ }^{23}$.

La dinastia Lehman, dunque, è modello di integrazione di una minoranza quella ebraica - emigrata per liberare l'imprenditorialità, senza dover subire discriminazioni ${ }^{24}$, profittando anzi di una forte identità comunitaria, perché capace di sfruttare quanto offerto da un Paese - gli Stati Uniti - ricco di materie prime, ma bisognoso di energie umane aperte allo sviluppo del capitalismo moderno. La narrazione drammaturgica di Massini, quindi, spettacolarizza un fenomeno (la predisposizione degli Ebrei, in particolare in ambito americano, al capitalismo), che nel caso specifico è stato oggetto di studi, soprattutto dopo la lettura datane dal premio Nobel 1976 per l'economia, Milton Friedman. Nel suo intervento Capitalism and the Jews, presentato per una conferenza alla Mont Pelerin Society nel $1972^{25}$, lo studioso americano parte da un paradosso, esponendo due tesi tra loro contraddittorie: da un lato gli Ebrei sono enormemente debitori verso il capitalismo occidentale per la libertà d'impresa di cui hanno goduto; dall'altro lato, nel '900 gli Ebrei si sono opposti al capitalismo cercando di minarlo ideologicamente. Esaminando il testo che all'inizio del xx secolo ha introdotto la questione, Die Juden und das Wirtschaftsleben di Werner Sombart ${ }^{26}$, Friedman individua un conflitto tra l'agire economico degli Ebrei - di maggior successo laddove la libertà imprenditoriale sia garantita senza preclusioni etniche - e gli ideali socialisti, a loro cari per due ragioni: nei Paesi dell'Europa

23. Massini Stefano, Lehman Trilogy, cit. p. 270; in Qualcosa sui Lehman (p. 718) diventa "Ora che i Lehman sono sangue americano/ chi se li ricorda i riti dell'Europa?".

24. Bisogna tuttavia ricordare che il fallimento della Lehman Brothers \& Co. nel settembre 2008 fece riemergere diffusi sentimenti anti-semiti (cfr., a titolo informativo, Greenberg Brad A., "Blaming the Jews...Again", Jewish Journal, 9 aprile 2009, https:/ / jewishjournal.com/uncategorized/69740/; e una recensione sulla messinscena a Broadway della primavera 2019, che stronca il testo di Massini: Miller Judith, "The Lehman Trilogy': Can a Jewish immigrant family embody the American dream?", Pundicity.com, 17 aprile 2019, http://www.judithmiller.com/22575/lehman-trilogy).

25. Friedman Milton, "Capitalism and the Jews", Foundation for Economic education, 1 ottobre 1988, https:/ / fee.org/articles/capitalism-and-the-jews/.

26. Leipzig, Duncker \& Humblot, 1911 (consultato nella sua traduzione inglese in ristampa anastatica: The Jews and the Modern Capitalism, traduzione di Martin Epstein, London, T. F. Unwin, 1913). 
occidentale, la loro emancipazione sociale e la piena fruizione della cittadinanza sono state favorite dalle forze politiche democratiche e progressiste; inoltre, l'ideale socialista fa sbiadire il tòpos antisemita della rapace avidità, presentando invece questo antico popolo come solidale e generoso.

In tempi recenti la questione posta da Friedman è stata affrontata dallo storico Jerry Z. Muller ${ }^{27}$ : gli Ebrei custodiscono una cultura plurimillenaria fondata sui mitzvot, i precetti, che istituiscono uno stile di vita basato sull'autodisciplina, la lucida pianificazione dell'agire (si ricordi l'ossessivo "controllo controllo controllo" di Philip Lehman nei testi di Massini), un uso parsimonioso delle risorse sia materiali sia fisiche, la soluzione non-violenta dei conflitti e la valorizzazione delle risorse intellettuali rispetto alla prodezza atletica. A questo retaggio nei secoli si è aggiunta la loro straordinaria capacità di mediazione, sia in campo mercantile sia nel tessere reti sociali dal forte valore fiduciario, tale da varcare $i$ ristretti confini comunitari e geografici.

Nell'attraversare il secolo pieno della modernità, il '900, la famiglia Lehman fornisce una concreta rappresentazione degli orizzonti economici globali e globalizzati - ben prima della caduta del muro di Berlino - e del loro principale supporto teorico, il postmodernismo, se per tale si intende, dal punto di vista economico, un mondo globale privo di un centro dominante, una società post-industriale (almeno nei Paesi occidentali a capitalismo avanzato, salvo sfruttare la delocalizzazione nei Paesi in via di sviluppo, spesso privi di tutele ambientali), e politicamente, il venir meno della lotta di classe, dovuto però non a un consenso condiviso e compartecipato - com'era nella mente dei teorici postmoderni - ma a una progressiva erosione, per non dire sparizione, delle classi sociali medesime ${ }^{28}$.

La scrittura di Massini è culturalmente postmoderna nel suo mescolare stili e nel multiple coding: nel capitolo XVII del romanzo, Golyat, dalla lettura di un trafiletto su Nietzsche, Bobbie Lehman ha l'idea di finanziare un fumetto che trasmetta ottimismo all'America del New Deal e anti-nazista, Superman; il testo meramente verbale visualizza in un improvviso excerptum di graphic nove ${ }^{9}$ l'aspirazione del magnate ebreo di salvare, novello Davide, sua moglie Ruth simbolo di tutta l'America - dal mostro Golyat, un gigante ipertrofico avvolto in

27. Muller Jerry Z., Capitalism and the Jews, Princeton, Princeton University Press, 2010 (in particolare il capitolo The Jewish Response to Capitalism. Milton Friedman's Paradox Reconsidered, pp. 72-132).

28. Cfr. Jencks Charles (a cura di), The Post-modern Reader, London-New York, Academy Editions-St. Martin's Press, 1992.

29. Massini Stefano, Qualcosa sui Lehman, cit., pp. 687-697. 
una tuta attillata sul cui torace campeggia una croce uncinata: si crea così - come scritto da Umberto Eco - quel mito ideale proprio di "una società industriale dove l'uomo diventa numero nell'ambito di una organizzazione che decide per lui; $[\ldots]$ in una società di tale tipo l'eroe positivo deve incarnare oltre ogni limite pensabile esigenze di potenza che il cittadino comune nutre e non può soddisfare" ${ }^{30}$. La famiglia Lehman è dunque l'esempio di come il capitalismo abbia plasmato l'immaginario collettivo nella sua fase di maggiore espansione, e di come il suo fallimento non sia stato meramente finanziario, ma anche culturale.

Nel 1990 Franco Fortini raccoglieva in un volumetto, Extrema Ratio, dal sottotitolo Note per un buon uso delle rovine, delle riflessioni influenzate dal crollo del comunismo in Europa orientale. In una di queste, dal titolo Un decennio di Postmoderno, l'intellettuale fiorentino si interroga sulla reale validità dell'affermata teoria e - soprattutto - sulla sua coerenza interna: il postmoderno ha contribuito sicuramente ad abbattere la cultura borghese, ma adotta la medesima struttura dialettica nell'interpretare la realtà:

L'età del postmoderno continua a porgerci ogni giorno segni e sintomi temibili. Questa nostra interpretazione diventa uno di quei segni. L'impero dei segni si rivela segno del potere imperioso. L'apparenza si afferma come unica realtà? Fingiamolo pure. Voler mutare le apparenze sarà dunque come voler mutare la realtà ${ }^{31}$.

Il postmoderno, come la parabola economica dei Lehman, pone il rapporto tra segno e senso: nel brulichio dei segni, come nel chiacchiericcio invadente che accoglieva i Lehman a New York dalla loro tranquilla Alabama, può celarsi la perdita di senso, se la "confusione delle forme e delle culture" 32 è comunque impostata su di una visione politica ed economica propria della società che in origine contesta. Per uscire dal postmoderno e dalla società in cui esso si è affermato, si deve elaborare una visione antitetica, capace di superare il tardo capitalismo, mai realmente abbandonato dalla crisi innescata nel 2008 dal fallimento della Lehman Brothers \& $\mathrm{Co}^{33}$.

L'invenzione epica-narrativa-drammatica di Massini fornisce uno strumento fondamentale, come espresso nel titolo di questa riflessione, per leggere i nostri

30. Eco Umberto, Apocalittici e integrati. Comunicazioni e teorie della cultura di massa, Milano, Bompiani, $1995^{3}$, p. 227.

31. Fortini Franco, Extrema Ratio, Milano, Garzanti, 1990, p. 86.

32. Ibid.

33. Sullo stravolgimento del regime di vita postmoderno causato dalla pandemia cfr. Mamzer Hanna, "Postmodern Society and Covid-19 Pandemic: Old, New and Scary", Society Register, vol 4, n. 2, pp. 13-16. 
giorni, ri-spettandoli e captandone un senso storico-emotivo, non meramente numerico. Ponendo attenzione al linguaggio adoperato sui social, sui media e nei discorsi comuni durante la segregazione forzata a scopo preventivo, e ancor più dopo l'allentamento di queste misure, si è notata l'abbondanza di un vocabolo - fondamentalmente -, 'normalità', centro di un campo semantico espanso e raggrumato dal prefisso $r i$-: $r i$-torno, $r i$-presa, $r$-cominciare, tanto per farne degli esempi (ma ci si può sbizzarrire al riguardo), esprimenti quindi un desiderio di archiviare - se non cancellare - nel minor tempo possibile l'impietosa esperienza che ha smascherato le reali distonie di un sistema sociale ed economico votato alla diffusione di un illusorio e mercificato wellness. Come ha scritto chiaramente Giovanni Nencioni, tale prefisso deriva dal "latino re- (o red-) che indicava un movimento a ritroso (recedere 'tirarsi indietro', redire 'ritornare') o il ritorno a uno stato anteriore (reficere 'riattare, riparare', restituere 'restaurare') o un movimento in senso contrario che distrugge ciò che è stato fatto (precludere 'aprire', revelare 'scoprire')" 34 . La diffusa volontà, dunque, di " $r i$ "” poco o nulla ha a che vedere con il coraggio imprenditoriale dei tre fratelli ebrei emigrati dalla Germania per partecipare liberamente al "carillon/ chiamato America"35, il calcolo lucido - ma non cinico - di Philip Lehman, l'irrequietezza onnivora di Dawid Lehman, energie di visionaria progettualità, ma è invece un à rebours astorico, alla ricerca di una nuova, ennesima esperienza di un rifugio edenico pur con tutte le sue storture, acriticamente benvenuto perché noto.

In una intervista rilasciata da Stefano Massini al quotidiano fiorentino $\mathrm{La}$ Nazione ${ }^{36}$ ancora lo scorso marzo, lo scrittore rilevava come ci si occupasse in modo pressoché esclusivo delle conseguenze economico-sociali della pandemia, e decisamente meno di quelle psico-emotive ${ }^{37}$, perché il virus - "minuscolo

34. Nencioni Giovanni, “Risposta 5”, La Crusca per voi, n. 4, aprile 1992.

35. Massini Stefano, Lehman Trilogy, cit., p. 9.

36. Giuliani Foti Titti, "Massini: 'Storie di vita dal nemico invisibile", La Nazione, 25 marzo 2020, https:/ / www.lanazione.it/firenze/cronaca/intervista-massini-1.5081350.

37. A dire il vero, tali conseguenze sono state oggetto di narrazioni e di analisi (ma suppositive, non potendo terapeuticamente rapportarsi direttamente con chi ne subisse un disagio emotivo): cfr. il documento istituzionale dell'Inter-Agency Standing Committee, organo delle Nazioni Unite creato nel 1991, Interim Briefing Note Addressing Mental Health and Psychosocial Aspects of COV ID-19 Outbreak. (developed by the IASC's Reference Group on Mental Health and Psychosocial Support), https://interagencystandingcommittee. org/iasc-reference-group-mental-health-and-psychosocial-support-emergencysettings/interim-briefing, pubblicato in traduzione dal nostro Ministero della Salute (http://www.salute.gov.it/imgs/C_17_pagineAree_5373_10_file.pdf), e il bell'articolo di De FAzıo Camilla, "La salute mentale ai tempi del coronavirus. Gli studi in Italia", quotidianosanità.it, 18 aprile 2020, https:/ /www.quotidianosanita.it/scienza-e-farmaci/ 
esserino invisibile" - nega le certezze fondamentali: lo spazio e il tempo (nella reclusione imposta per legge), ma soprattutto la fiducia nel noto, perché il contagio arriva proprio dalla prossimità.

Da quest'ultimo valore - la prossimità: conoscere realmente la cronotopia circostante - scaturisce il $r$-spettare: voltarsi indietro a scrutare (re-spicio) con sguardo indagatore, e anche riguardare, perché è una memoria che non vogliamo abbandonare. La narrazione epico-tragica di Stefano Massini, dunque, ricorda quanto importante, nell'attraversare la Storia, resti la verbalizzazione delle emozioni in chiave - questa sì - realmente postmoderna, perché non catartica, rinunciando al "fatidico messaggio": "non avremo in premio assoluzioni, condanne, né tantomeno manuali di condotta"38.

Maurizio ReBAudengo

Convitto Nazionale "Umberto I" - Torino

articolo.php?articolo_id $=84201$. Nel dibattito, privilegiato è stato l'ambito educativodidattico, che ha obbligato docenti e discenti a stravolgere di punto in bianco le modalità del rapporto e della fruizione del medesimo (si veda, a mo' di esempio, il blog 'Diario da una quarantena", pubblicato su griseldaonline: https://site.unibo.it/griseldaonline/ it/diario-quarantena); cfr. anche Stankovska Giordana, Memedi Imran, Dimitrovski Dimitar, "Coronavirus Covid-19 Disease, Mental Health and Psychosocial Support", Society Register 2020, cit., pp. 33-47.

38. Ronconi Luca, Prefazione, cit., pp. VI-VII. 